\title{
Clinicopathologic Characteristics and Causes of Postmenopausal Bleeding in Older Patients
}

\author{
Hyen Chul Jo ${ }^{1}$, Jong Chul Baek ${ }^{1}$, Ji Eun Park ${ }^{1}$, Ji Kwon Park ${ }^{1}$, In Ae Cho ${ }^{2}$, Won Jun Choi ${ }^{2}$, Joo Hyun Sung ${ }^{3}$ \\ ${ }^{1}$ Department of Obstetrics and Gynecology, Gyeongsang National University Changwon Hospital, Gyeongsang National University, Changwon, \\ Korea \\ ${ }^{2}$ Department of Obstetrics and Gynecology, Gyeongsang National University Hospital, Gyeongsang National University, Jinju, Korea \\ ${ }^{3}$ Department of Occupational and Environmental Medicine, Gyeongsang National University Changwon Hospital, Gyeongsang National \\ University, Changwon, Korea
}

Corresponding Author:

Jong Chul Baek, MD, $\mathrm{PhD}$

https://orcid.org/0000-0003-0919-0800

Department of Obstetrics and

Gynecology, Gyeongsang National

University Changwon Hospital,

11, Samjeongja-ro, Seongsan-gu,

Changwon 51472, Korea

Tel: +82-55-214-3781

Fax: +82-55-759-1118

E-mail: gmfather@gmail.com

Received: October 25, 2018

Revised: November 21, 2018

Accepted: November 23, 2018

\begin{abstract}
Background: This study aimed to reveal the clinicopathologic features and causes of bleeding in older patients with postmenopausal bleeding (PMB) and to investigate the correlation between the ultrasonographic findings and etiology of PMB. Methods: We retrospectively analyzed the causes and clinical characteristics of PMB in 498 patients who were diagnosed between January 2007 and December 2017. The population with PMB was divided into 2 groups according to age: Group A $(n=204)$ included individuals more than 65 years of age and group B $(n=294)$ included those less than 65 years of age. Clinical characteristics such as age, parity, underlying conditions, previous surgical history, and previous menopausal hormone therapy were compared between the groups. Cervical cytology testing and transvaginal ultrasonography were performed in all patients with PMB. Endometrial biopsy was performed in all cases of endometrial thickness $\geq 5 \mathrm{~mm}$. Results: We examined 498 patients with PMB. In group A, atrophic endometrium $(n=125,61.27 \%)$ was the most common cause of PMB. Twenty-three patients had gynecological malignancy (cervical cancer: $n=12,5.88 \%$; endometrial cancer: $n=8,3.42 \%$; ovarian cancer: $n=3$, $1.46 \%$ ), and 30 patients had benign gynecological disease (endometrial polyp: $n=10$, 4.90\%; submucosal myoma: $n=6,2.94 \%$; uterine prolapse: $n=7,3.42 \%$; cervical dysplasia; $n=5,2.45 \%$; cervical polyp: $n=2,0.98 \%$ ). Forty patients had endometrial thickness $\geq 5 \mathrm{~mm}$. Eight patients were diagnosed with endometrial cancer. All cases of endometrial cancer were diagnosed with endometrial thickness $>10 \mathrm{~mm}$. Conclusion: Atrophic endometrium was the most common cause of PMB in both groups, and approximately $12 \%$ of cases were associated with gynecological malignancy in older patients. (Ann Geriatr Med Res 2018;22:189-193)
\end{abstract}

Key Words: Postmenopause, Vaginal bleeding, Elderly

\section{INTRODUCTION}

Postmenopausal bleeding (PMB) is a common condition with a frequency as high as 10\% among menopausal women. ${ }^{1,2)}$ The most common causes of PMB are benign diseases such as vaginal or endometrial atrophy, hormone replacement therapy, endometrial or cervical polyps, and other miscellaneous diseases. However, other causes include malignant gynecological diseases such as cervical cancer, endometrial cancer, and uterine sarcoma. ${ }^{3)}$ In the diagnostic evaluation of older patients with PMB, it is important to exclude gynecological malignancy.

Ten percent of all women with endometrial cancer present with PMB. ${ }^{4,5}$ Transvaginal ultrasonography (TVS) is widely used as a diagnostic modality in evaluating the endometrium of women with PMB. When endometrial thickness (ET) is $5 \mathrm{~mm}$ or more, or ET cannot be sufficiently evaluated, other investigations are warranted, such as endometrial sampling and/or hysteroscopy. Curettage biopsies are a method of choice in differential diagnosis of endometrial pathology.

The incidence of cervical cancer has been steadily decreasing in South Korea. However, cervical cancer is the most common type of gynecological cancer, and a common cause of PMB. Cervical cytology testing should therefore be performed in all PMB patients. Previous studies have identified the causes of PMB in menopausal women. However, there is a lack of data regarding the causes of PMB in older patients. Clinicopathologic features and causes in older individuals differ from those of patients who experience PMB immediately after menopause. The objective of this study should be here. 


\section{MATERIALS AND METHODS}

From January 2007 to December 2017, we retrospectively analyzed 533 patients with PMB who visited the Department of Obstetrics and Gynecology in Gyeongsang National Univeristy Hosptial via outpatient clinic or the Emergency Department. The Korean Standard Classification of Diseases 7th edition that modified the International Statistical Classification of Diseases and Related Health Problems 10th edition was used for diagnostic specification. The definition of gynecologic diseases was based on the diagnostic codes for vaginal bleeding (N939), cervical cancer (C53.x), endometrial cancer (C54.x), ovarian cancer (C56.x), endometrial hyperplasia (N85.x), endometrial polyp (N84.x), cervical polyp (N84.x), pyometra (N71.x), myoma (D25.x), and cervical intraepithelial neoplasia (N87.x). The study population was divided into 2 groups according to age: one group included those more than 65 years of age (group A), and the other included those less than 65 years of age (group B). Among the patients analyzed, 498 were evaluated for causes of PMB, after excluding patients with a history of hysterectomy due to gynecological problems $(n=21)$, systemic and hematological diseases $(n=6)$, trauma $(n=5)$, or those were undergoing radiation therapy for cervical cancer $(n=4)$. Group A comprised 204 patients, and group B comprised 294 patients. Data were grouped into the following categories: age at diagnosis, parity, underlying conditions, previously prescribed medications, nongynecological surgical history, gynecological surgical history, and ET measured by TVS.

TVS and cervical cytology testing were performed in all patients with PMB. TVS was the initial examination performed to evaluate the endometrium in older patients. Individuals who were found to have an ET equal to or greater than $5 \mathrm{~mm}$ underwent endometrial sampling with cervical dilatation and curettage (D\&C) under local anesthesia. In cases of contraindication to local anesthesia, official D\&C biopsy was conducted without anesthesia. If endometrial biopsy did not provide sufficient histological information for diagnosis, hysteroscopy was also performed. To determine the status of the uterine cervix, cervical cytology screening was conducted; if this testing yielded abnormal results, colposcopic cervical biopsy was performed. Pyometra was defined as pus collection in the endometrial cavity in the absence of abnormal results from endometrial histology and cervical cytology.

Statistical analysis was conducted with IBM SPSS Statistics ver. 24.0 (IBM Co., Armonk, NY, USA), and a pvalue of less than 0.05 was considered significant. Nominal variables were expressed as number (n) and \%, and continuous variables were expressed as median (range) or mean \pm standard deviation. The Student t-test was used to calculate the p-value for continuous variables, and the chi-square test was used to calculate the p-value for nominal variables.

\section{Ethical Statement}

Because the present study used data that could not be personally identified, it was exempt from Institutional Review Board review according to the Korean Bioethics and Safety Act.

\section{RESULTS}

\section{Patient Characteristics}

The study population comprised 498 patients (Table 1). Clinical characteristics of group A were as follows: age distribution, 65 to 98 years; average age, $74 \pm 7.12$ years; parity, $3.7 \pm 1.58$. Among the patients in this group, numerous patients exhibited underlying conditions, such as hypertension, diabetes mellitus, cerebrovascular disease, or lung cancer, found in 78, 45, 18, and 4 patients, respectively. Forty-five patients had 2 underlying conditions, and 6 had 3 or more underlying conditions. The most common nongynecological surgery undergone by patients was that for colon cancer, and gynecological operations included cesarean section, tubal ligation, and salpingectomy owing to ectopic pregnancy.

\section{Causes of Bleeding}

Table 2 shows the causes of PMB in the 2 groups. The most common cause of PMB was atrophic endometrium in both groups. In group A, in the present study, 125 patients (61.27\%) were diagnosed with atrophic endometrium. In

Table 1. General patient characteristics

\begin{tabular}{|c|c|c|c|}
\hline Characteristic & $\begin{array}{l}\text { Group A } \\
(n=204)\end{array}$ & $\begin{array}{l}\text { Group B } \\
(n=294)\end{array}$ & $\mathrm{p}$-value \\
\hline Age (yr) & $74.10 \pm 7.12$ & $57.04 \pm 3.81$ & $<0.001$ \\
\hline Parity & $3.71 \pm 1.59$ & $2.27 \pm 0.97$ & $<0.001$ \\
\hline \multicolumn{4}{|l|}{ Underlying condition } \\
\hline \multicolumn{4}{|l|}{ Cardiovascular disease } \\
\hline Hypertension & $78(38.24)$ & $56(19.05)$ & $<0.001$ \\
\hline Ischemic heart disease & $5(2.45)$ & $9(3.06)$ & 0.685 \\
\hline Arrhythmia & $4(1.96)$ & $3(1.02)$ & 0.381 \\
\hline \multicolumn{4}{|l|}{ Endocrine disease } \\
\hline Diabetes mellitus & $45(22.06)$ & $26(8.84)$ & $<0.001$ \\
\hline Thyroid disease & $2(0.98)$ & $7(2.38)$ & 0.249 \\
\hline Cerebrovascular disease & $18(8.82)$ & $9(3.06)$ & 0.005 \\
\hline \multicolumn{4}{|l|}{ Anticoagulant usage } \\
\hline Aspirin & $23(11.27)$ & $23(7.82)$ & 0.191 \\
\hline Warfarin & $12(5.88)$ & $8(2.72)$ & 0.077 \\
\hline \multicolumn{4}{|c|}{ Nongynecologic surgical history } \\
\hline Breast cancer & $2(0.98)$ & $23(7.82)$ & 0.001 \\
\hline Thyroid & $6(2.94)$ & $14(4.76)$ & 0.309 \\
\hline Colon cancer & $10(4.90)$ & $5(1.70)$ & 0.040 \\
\hline Lung cancer & $4(1.96)$ & $4(1.36)$ & 0.600 \\
\hline Brain & $2(0.98)$ & $2(0.68)$ & 0.712 \\
\hline
\end{tabular}

Values are presented as mean \pm standard deviation or number (\%). Group A, postmenopausal bleeding (PMB) patients over 65 years old; group B, PMB patients less than 65 years old. 
Table 2. Etiology of postmenopausal bleeding

\begin{tabular}{lccc}
\hline \multicolumn{1}{c}{ Disease } & $\begin{array}{c}\text { Group A } \\
(\mathrm{n}=204)\end{array}$ & $\begin{array}{c}\text { Group B } \\
(\mathrm{n}=294)\end{array}$ & p-value \\
\hline Atrophic endometrium & $125(61.27)$ & $156(53.06)$ & 0.069 \\
Pyometra & $17(8.33)$ & $7(2.38)$ & 0.002 \\
Cervical cancer & $12(5.88)$ & $31(10.54)$ & 0.069 \\
Endometrial polyp & $10(4.90)$ & $7(2.38)$ & 0.128 \\
\hline Endometrial cancer & $8(3.92)$ & $19(6.46)$ & 0.218 \\
Uterine prolapse & $7(3.43)$ & - & 0.001 \\
Submucosal myoma & $6(2.94)$ & $13(4.42)$ & 0.396 \\
Cervical dysplasia & $5(2.45)$ & $14(4.76)$ & 0.186 \\
Anticoagulant usage & $5(2.45)$ & $4(1.36)$ & 0.369 \\
Ovarian cancer & $3(1.47)$ & - & 0.037 \\
Menopausal hormone therapy & $3(1.47)$ & $24(8.16)$ & 0.001 \\
Cervical polyp & $2(0.98)$ & $14(4.76)$ & 0.019 \\
Endometrial hyperplasia & - & $5(1.70)$ & 0.061 \\
Mesh erosion & $1(0.49)$ & - & 0.219 \\
\hline
\end{tabular}

Values are presented as number (\%).

Group A, postmenopausal bleeding (PMB) patients over 65 years old; group B, PMB patients less than 65 years old.

addition, there were 17 cases of pyometra (8.33\%), 12 cases of cervical malignancy (5.88\%), 10 cases of endometrial polyp (4.90\%), 8 cases of endometrial cancer (3.92\%), 7 cases of uterine prolapse (3.42\%), 6 cases of submucosal myoma (2.94\%), 5 cases of cervical dysplasia (2.45\%), 5 cases of anticoagulant usage (2.45\%), 3 cases of menopausal hormone therapy (MHT) (1.46\%), 3 cases of ovarian cancer (1.46\%), 2 cases of cervical polyp (0.98\%), and 1 case of mesh erosion (0.49\%). Among the 5 cases of cervical dysplasia, 2 patients had moderate dysplasia and 3 had severe dysplasia. In the anticoagulant medication group, aspirin was the most common medication, followed by warfarin. In 2 out of the 5 cases in which bleeding occurred due to anticoagulant medication, the women used warfarin as therapy for atrial fibrillation. The single case of mesh erosion was due to a foreign body reaction to a mesh used in an operation for stress incontinence. Pyometra, uterine prolapse, and ovarian malignancy demonstrated a statistically significantly higher incidence in group A. MHT and cervical polyps showed a higher incidence in group B.

\section{ET and Histological Findings}

ET was measured by TVS in all 204 older patients. Table 3 shows the incidence of PMB stratified according to ET. The average ET was $3.71 \pm 4.0 \mathrm{~mm}$. The numbers of patients with $\mathrm{ET}<5 \mathrm{~mm} ; 5 \leq \mathrm{ET}<10 \mathrm{~mm}$; and ET $\geq 10 \mathrm{~mm}$ were 164,19 , and 16, respectively. Nineteen patients had an ET between 5 and $10 \mathrm{~mm}$; 3 among these presented with endometrial polyps. In contrast, in patients with ET greater than $10 \mathrm{~mm}, 8$ had endometrial cancer, 3 had endometrial polyps, and 5 had submucosal myoma. All patients with endometrial cancer had ET>10 mm. Five patients had mass-like lesions in the endometrium, with mass sizes of 7 , 13, 20, 35, and $50 \mathrm{~mm}$, respectively, and an average size
Table 3. Distribution of causes according to sonographic characteristics in group A

\begin{tabular}{|cc}
\hline Sonographic characteristic & No. of patients \\
\hline Endometrial thickness & \\
$\leq 5 \mathrm{~mm}$ & 164 \\
$\geq 5 \mathrm{~mm}, \leq 10 \mathrm{~mm}$ & 19 \\
$\geq 10 \mathrm{~mm}$ & 16 \\
\hline Endometrial polyp & 3 \\
\hline Endometrial cancer & 8 \\
\hline Submucosal myoma & 5 \\
\hline Endometrial polyp & 3 \\
\hline Mass & 5 \\
\hline Endometrial polyp & 4 \\
\hline Submucosal myoma & 1 \\
\hline
\end{tabular}

Group A, postmenopausal bleeding patients over 65 years old.

of $25 \pm 17.44 \mathrm{~mm}$. Four of these patients had endometrial polyps, and 1 had submucosal myoma (mass size, $35 \mathrm{~mm}$ ).

\section{DISCUSSION}

This study evaluated the common causes of PMB in menopausal patients. Among group A patients with PMB, atrophic endometrium was the most common cause (125 cases), followed by pyometra, cervical cancer, endometrial polyp, endometrial cancer, uterine prolapse, submucosal myoma, cervical dysplasia, anticoagulant usage, ovarian cancer, MHT, cervical polyp, and mesh erosion. These findings differed from those in group B. While the leading cause of PMB in this group was the same (atrophic endometrium), the rank order of the other causes differed. The prevalence rates of atrophic endometrium in groups A and B were $61.27 \%$ and 53.06\%, respectively. Atrophic endometrium was the most common cause of PMB and was reported in approximately 59\% of cases. ${ }^{6)}$ MHT as a cause of PMB showed a significantly different prevalence of $1.46 \%$ in group A and 8.16\% in group B. The prevalence of PMB caused by gynecological malignancy did not significantly differ between the 2 groups, at $11.26 \%$ and $16.96 \%$ in groups A and B, respectively.

PMB caused by cervical pathological abnormalities in group A was observed in 19 cases (9.31\%). Among these, there were 12 cases of cervical malignancy (5.88\%), 5 of cervical dysplasia (2.45\%), and 2 of cervical polyps (0.98\%). Cervical cancer was the most common cause of gynecologic malignancy resulting in PMB in both groups. The incidence rates of cervical cancer in groups $\mathrm{A}$ and $\mathrm{B}$ were $5.88 \%(n=12)$ and $10.54 \%(n=31)$, respectively. There was no statistically significant difference between the 2 groups. Both the incidence and mortality rates of cervical malignancy in South Korea have been declining. This decrease is due to the national cervical cancer screening program, which employs cervical cytology, as well as the nation's improved socioeconomic status, and also results from the fact that early diagnosis allows timely treatment. However, 
the incidence of cervical malignancy in South Korea remains higher than that in other Western countries.

In the present study, the prevalence of endometrial cancer was 5.19\% $(n=8)$ in group A. The numbers of cases of PMB caused by endometrial cancer in groups A and B were 8 (3.92\%) and 19 (6.46\%), respectively. For women with PMB who do not use MHT, the incidence of endometrial malignancy ranges from $4.9 \%$ to $11.5 \%{ }^{6,7)}$ In differential diagnosis of endometrial malignancy in women with PMB, TVS should be used to measure the thickness and shape of the endometrium. TVS is the most commonly used method for women with PMB because it is both noninvasive and effective. Thicker ET is associated with a greater likelihood of endometrial pathologies such as endometrial cancer, hyperplasia, and polyps. A number of cutoff values for ET have been suggested to determine whether endometrial sampling is required. An optimal cutoff of $5 \mathrm{~mm}$ is currently proposed. ${ }^{89)}$ There is little benefit to performing endometrial sampling in cases of ET less than $5 \mathrm{~mm}$.

In group A, 40 patients (19.6\%) exhibited ET greater than $5 \mathrm{~mm}$. Among these, 24 patients (11.76\%) were diagnosed with endometrial abnormality, 10 had endometrial polyps, 8 exhibited submucosal myoma, and 8 had endometrial malignancy. Diagnosis of endometrial polyps and submucosal myoma may be difficult with TVS and endometrial sampling alone. In such cases, hysteroscopy offers good diagnostic accuracy for structural lesions, such as polyps and leiomyoma, because it permits visual confirmation and endometrial biopsy during the procedure. Hysteroscopy can be performed in an office setting with local or no anesthesia, with or without sedation, factors offering a significant advantage to older patients. In the present study, endometrial cancer was diagnosed in 8 patients. The average ET in patients with endometrial cancer was $12.75 \pm 2.65 \mathrm{~mm}$. All patients who were diagnosed with endometrial cancer in group A had ET>10 mm. Another study reported that ET $<5 \mathrm{~mm}$ was associated with a $4 \%$ chance of endometrial cancer. ${ }^{10)}$ However, we did not perform endometrial sampling in patients with $\mathrm{ET}<5 \mathrm{~mm}$ in the current study.

Pyometra is an uncommon condition defined by the accumulation of purulent material in the uterine cavity. The condition can present with symptoms such as purulent vaginal discharge, PMB, and lower abdominal pain. However, most cases are asymptomatic with nonspecific symptoms. In the present study, 17 patients (8.33\%) in group A were diagnosed with pyometra as the cause of PMB. However, this diagnosis was indicated as the cause of PMB in 7 patients $(2.38 \%)$ in group B, indicating a significant difference in incidence. All patients in group B were older than 61 years. Pyometra is reported to occur between the ages of 34 and 92 years, and the median age is 75 years. ${ }^{11)}$ Additionally, pyometra is found in less than 1\% of individuals in the general gynecologic population, and its incidence in older patients is approximately $13.6 \%{ }^{12)}$ Along with age, underlying diseases such as diabetes are also a risk factor for pyometra. Approximately 22\%-77\% of pyometra cases are associated with malignant tumors such as cervical cancer, endometrial cancer, and rectal cancer. ${ }^{13,14)}$ Therefore, examination using TVS, cervical cytology, and endometrial sampling should be performed to exclude malignancy.

MHT was another cause of PMB in the present study. About 50\% of women using continuous estrogen-progestin combined replacement regimens experience vaginal bleeding within 6 months after initiation of therapy. ${ }^{15)}$ In this study, 5 patients in group A received MHT. Among these patients, 2 were diagnosed with endometrial cancer, and $3(1.46 \%)$ had atrophic endometrium. In group B, 24 patients $(8.16 \%)$ were diagnosed with PMB caused by MHT. In the present study, a significant difference in prevalence among the causes of PMB between the groups was observed with MHT. This difference may be because MHT is usually used in the short term, within 5 years after menopause, and is not recommended in older individuals.

Three of the patients with PMB in group A were diagnosed with ovarian malignancy. Among these, 2 patients had serous cystadenocarcinoma, and 1 had clear cell carcinoma. Ovarian cancer usually presents with nonspecific symptoms such as abdominal pain and increased abdominal size. Vaginal bleeding is a rare symptom in epithelial ovarian cancer, which is associated with sex cord-stromal tumors such as granulosa cell tumors. The incidence of ovarian cancer increases with advancing age, and symptoms do not develop until the cancer is at an advanced stage. In older patients with vaginal bleeding, it may be useful to survey the endometrium as well as pelvic structures, including the bilateral ovaries, during TVS.

One patient in the current study experienced vaginal bleeding due to a foreign body reaction. This reaction was the result of erosion of the mesh used in stress urinary incontinence surgery. Bleeding was checked in the area of erosion and no abnormal findings other than inflammation were observed on gynecological examination. The incidence of mesh erosion following surgery for stress urinary incontinence varies widely from $0 \%$ to $7.3 \%$. $^{16,17)}$ Advanced age, diabetes mellitus, smoking, and previous vaginal surgery for pelvic organ prolapse or incontinence are associated with increased risk of mesh erosion. It is important to perform a gynecological examination and to review the patient's surgical history in cases of vaginal bleeding, because surgery for stress urinary incontinence is becoming a more common procedure.

Vaginal bleeding in postmenopausal women may arise from several nongynecological causes such as hematuria and hematochezia. If the cause of bleeding cannot be identified through gynecological examination and other methods, patients should be referred to urology or general surgery. When evaluating patients with PMB, the clinician should consider all these possibilities.

The present study had several limitations. First, this 
study was performed at a single tertiary medical center and involved a small number of patients. Hospital-based studies invariably tend to have falsely elevated incidence rates. Second, this retrospective study could not investigate other confounders, including the use of MHT in some patients, supplementation agents for underlying conditions, and social habits (e.g., diet, nutritional supplements, and exercise). Third, the sample size was too small to allow definite conclusions. Further population-based studies may prove valuable to compare PMB etiology and clinicopathologic characteristics. Another limitation of the present study is that endometrial biopsy was not performed in women with ET less than $5 \mathrm{~mm}$.

In conclusion, atrophic endometrium is the most common cause of PMB, and approximately 12\% of PMB cases among older patients are associated with gynecological malignancies such as cervical cancer, ovarian cancer, and endometrial cancer. Physicians should be aware of the possibility of malignancy in patients with PMB, and should conduct a thorough differential diagnosis. Endometrial biopsy should be performed to diagnose endometrial cancer and other gynecological diseases in older women with PMB with ET>5 mm or mass-like ultrasonographic findings.

\section{CONFLICTS OF INTEREST DISCLOSURES}

The researchers claim no conflicts of interest.

\section{REFERENCES}

1. Breijer MC, Mol BW. Transvaginal ultrasound measurement of the endometrium remains the first line test for investigating postmenopausal bleeding but integration of patient characteristics into testing may further improve diagnostic algorithms. BJOG 2016;123:447.

2. Salman MC, Bozdag G, Dogan S, Yuce K. Role of postmenopausal bleeding pattern and women's age in the prediction of endometrial cancer. Aust N Z J Obstet Gynaecol 2013;53:484-8.

3. Berek JS, Rinehart RD, Novak E. Berek \& Novak's gynecology. 15th ed. Philadelphia (PA): Wolters Kluwer Health/Lippincott Williams \& Wilkins; 2012.

4. Rose PG. Endometrial carcinoma. N Engl J Med 1996;335:640-9.

5. Raouf SA, Gupta P, Papaioannou S, Pradhan P. Endometrial thickness for invasive investigations in women with postmenopausal bleeding. Climacteric 2011;14:117-20.

6. Burbos N, Musonda P, Giarenis I, Shiner AM, Giamougiannis P, Morris E, et al. Age-related differential diagnosis of vaginal bleeding in postmenopausal women: a series of 3047 symptomatic postmenopausal women. Menopause Int 2010;16:5-8.

7. Ferrazzi E, Torri V, Trio D, Zannoni E, Filiberto S, Dordoni D. Sonographic endometrial thickness: a useful test to predict atrophy in patients with postmenopausal bleeding. An Italian multicenter study. Ultrasound Obstet Gynecol 1996;7:315-21.

8. Dueholm M, Marinovskij E, Hansen ES, Møller C, Ørtoft G. Diagnostic methods for fast-track identification of endometrial cancer in women with postmenopausal bleeding and endometrial thickness greater than $5 \mathrm{~mm}$. Menopause 2015;22:616-26.

9. Dueholm M, Hjorth IM, Secher P, Jørgensen A, Ørtoft G. Reproducibility of endometrial pathologic findings obtained on hysteroscopy, transvaginal sonography, and gel infusion sonography in women with postmenopausal bleeding. J Minim Invasive Gynecol 2015;22:1036-44.

10. Smith-Bindman R, Kerlikowske K, Feldstein VA, Subak L, Scheidler J, Segal M, et al. Endovaginal ultrasound to exclude endometrial cancer and other endometrial abnormalities. JAMA 1998;280:1510-7.

11. Yildizhan B, Uyar E, Sişmanoğlu A, Güllüoğlu G, Kavak ZN. Spontaneous perforation of pyometra. Infect Dis Obstet Gynecol 2006;2006:26786.

12. Geranpayeh L, Fadaei-Araghi M, Shakiba B. Spontaneous uterine perforation due to pyometra presenting as acute abdomen. Infect Dis Obstet Gynecol 2006;2006:60276.

13. Agarwal R, Suneja A, Sharma A, Vaid NB. An unusual etiology of spontaneous pyometra perforation; a case report. J Reprod Infertil 2011;12:235-8.

14. Cho IA, Park JE, Baek JC. Pyometra in elderly patients and its clinical characteristics. J Korean Geriatr Soc 2016;20:42-8.

15. Ettinger B, Li DK, Klein R. Unexpected vaginal bleeding and associated gynecologic care in postmenopausal women using hormone replacement therapy: comparison of cyclic versus continuous combined schedules. Fertil Steril 1998;69:865-9.

16. Guerrero KL, Emery SJ, Wareham K, Ismail S, Watkins A, Lucas MG. A randomised controlled trial comparing TVT, Pelvicol and autologous fascial slings for the treatment of stress urinary incontinence in women. BJOG 2010;117:1493-502.

17. Kennelly MJ, Moore R, Nguyen JN, Lukban JC, Siegel S. Prospective evaluation of a single incision sling for stress urinary incontinence. J Urol 2010;184:604-9. 